\title{
A NONMOVABLE SPACE WITH MOVABLE COMPONENTS
}

\author{
J. DYDAK, J. SEGAL AND S. SPIEŻ
}

(Communicated by Dennis Burke)

\begin{abstract}
In this paper we construct a nonmovable complete metric space of which every component is movable. This construction is based on P. Roy's famous example of a complete metric space $X$ which has inductive dimension ind $X=0$ and covering dimension $\operatorname{dim} X=1$.
\end{abstract}

Introduction. K. Borsuk [1, Theorem 7.1] proved that if every component of a compactum $X$ is movable, then $X$ is movable. In this paper we construct a nonmovable complete metric space every component of which is movable. The construction is based on P. Roy's famous example [5] of a complete metric space $X$ which has inductive dimension ind $X=0$ and covering $\operatorname{dimension} \operatorname{dim} X=1$.

Actually for any metric space $X$ with $\operatorname{dim} X=1$ we construct in $\S I-I X$ a related solenoidal-type space $Z$ and a morphism $\mathbf{p}=\left\{p_{n}\right\}$ from $Z$ into inverse system of the dyadic solenoid. We show that $p_{1}: Z \rightarrow S^{1}$ induces a nontrivial homomorphism on the first Čech cohomology $H^{1}(p): H^{1}\left(S^{1}\right) \rightarrow H^{1}(Z)$ and that this implies $Z$ is nonmovable. So in the case $X$ is Roy's example we get that $Z$ is nonmovable but each component is movable (each component is a point). The reader is referred to Spanier [6] as a general reference for the Hopf theorem.

We also present a second example in $§ \mathrm{XI}$ which is a totally disconnected, nonmovable space. This example is easier to construct but fails to have inductive dimension 0 and to be completely metrizable like the solenoidal Roy example mentioned above.

We were led to this question by a paper of M. A. Moron [4]. He showed that at least one of three results in shape theory for compacta did not carry over to the noncompact case. However, he could not ascertain which one failed in the noncompact case.

I. Construction of a solenoidal space. Let $X$ be a metric space with the covering dimension $\operatorname{dim} X=1$. There exists a closed subset $A \subset X$ and a map $f:(X, A) \rightarrow(I, \dot{I})$ that induces a nontrivial homomorphism $H^{1}(f): H^{1}(I, \dot{I}) \rightarrow$ $H^{1}(X, A)$. Let $f_{i}:\left(X_{i}, A_{i}\right) \rightarrow(I, \dot{I}), i=0,1$, be two copies of $f$. We denote by $Y$ the space obtained from the topological sum of $X_{0}$ and $X_{1}$ by the natural identification of $f_{0}^{-1}(1)$ and $f_{1}^{-1}(1)$. We assume that $X_{0}$ and $X_{1}$, and so also $B_{0}=f_{0}^{-1}(0), B_{1}=f_{1}^{-1}(0)$ and $B=B_{0} \cup B_{1}$ are subsets of $Y$. One can prove that the map $g:(Y, B) \rightarrow(I, \dot{I})$, given by

$$
g(y)= \begin{cases}\frac{1}{2} f_{0}(y) & \text { for } y \in X_{0} \\ 1-\frac{1}{2} f_{1}(y) & \text { for } y \in X_{1}\end{cases}
$$

Received by the editors January 28, 1987.

1980 Mathematics Subject Classification (1985 Revision). Primary 54F43; Secondary 54F45.

Key words and phrases. Movability, component, dimension, and shape. 
induces a nontrivial homomorphism $H^{1}(g): H^{1}(I, \dot{I}) \rightarrow H^{1}(Y, B)$. Let $s: Y \rightarrow Y$ be the symmetry function that assigns to a point $x \in X_{0}$ the corresponding point $s(x) \in X_{1}$. There is a homeomorphism $h$ defined on the Cantor set $C$ onto itself such that the quotient space $C \times I /(h(c), 0) \sim(c, 1)$, where $c \in C$, is the dyadic solenoid. We define a quotient space

$$
Z=C \times Y /(h(c), b) \sim(c, s(b)),
$$

where $c \in C$ and $b \in B_{0}$. Let $h: C \times Y \rightarrow C \times Y$ be defined by $h(c, y)=(h(c), y)$ for any $c \in C$ and $y \in Y$.

II. Now we will describe a morphism $\mathbf{p}=\left\{p_{n}\right\}$ from $Z$ into the dyadic solenoid's inverse system $S^{1} \stackrel{2}{\leftarrow} S^{1} \stackrel{2}{\leftarrow} S^{1} \leftarrow \cdots$ (see [3, p. 122]).

Let $\left\{C_{n}\right\}_{n=0}^{\infty}$ be the standard system of open coverings of the Cantor set $C$, i.e., $C_{n}=\left\{C_{n}^{k} \mid k=1, \ldots, 2^{n}\right\}$ consists of nonempty, open, pairwise disjoint subsets of $C$, each element of $C_{n}$ (a copy of $C$ ) contains exactly two elements of $C_{n+1}$ and $\left\{C_{n}\right\}_{n=0}^{\infty}$ is cofinal in the family of all open coverings of $C$. W may assume that the homomorphism $h$ induces a cyclic permutation on the set $C_{n}, h\left(C_{n}^{k}\right) \in C_{n}$ for each $C_{n}^{k} \in C_{n}, n>0$.

The set $Z$ is the sum of $2^{n}$ copies of $C_{n}^{k} \times Y$. The (continuous) map $p_{n}: Z \rightarrow S^{1}$ restricted to $C_{n}^{k} \times Y$ is the composition of the projection $C_{n}^{k} \times Y \rightarrow Y$, the map $g: Y \rightarrow I$ and an inclusion $i_{k}: I \rightarrow S^{1}$ ( $2^{n}$ segments $i_{k}(I)$ have disjoint interiors). We may assume that for each $n$ the following diagram commutes:

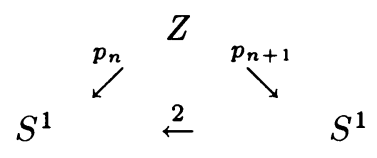

III. We will prove that $p=p_{1}: Z \rightarrow S^{1}$ induces a nontrivial homomorphism $H^{1}(p): H^{1}\left(S^{1}\right) \rightarrow H^{1}(Z)$. Let $Z=Z_{1} \cup Z_{2}$, where $Z_{i}$ is the image of $C_{1}^{i} \times Y$, where $C_{1}^{i} \in C_{1}$, under the projection $C \times Y \rightarrow Z$. Let $T, T_{0}$, and $T_{1}$ be the images of $C \times B, C_{1}^{1} \times B_{0}$, and $C_{1}^{1} \times B_{1}$, respectively, under this projection. So $T=Z_{1} \cap Z_{2}$. The map $p: Z \rightarrow S^{1}$ maps $Z_{i}$ onto a segment $I_{i} \subset S^{1}$. The intersection $I_{1} \cap I_{2}$ is $S^{0}$ (a two-point set). Let us consider the following diagram:

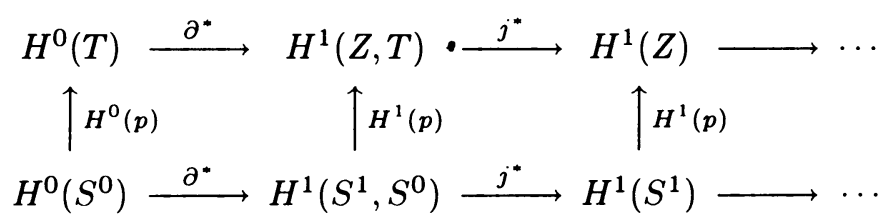

induced by the map $p$. By the Mayer-Vietoris sequence we can identify $H^{1}(Z, T)$ with $H^{1}\left(Z_{1}, T\right) \oplus H^{1}\left(Z_{2}, T\right)$ and $H^{1}\left(S^{1}, S^{0}\right)$ with $H^{1}\left(I_{1}, S^{0}\right) \oplus H^{1}\left(I_{2}, S^{0}\right)$ (the isomorphisms are induced by inclusions). Then $H^{1}(p): H^{1}\left(S^{1}, S^{0}\right) \rightarrow H^{1}(Z, T)$ we can identify with $H^{1}\left(p \mid Z_{1}\right) \oplus H^{1}\left(p \mid Z_{2}\right)$, where $H^{1}\left(p \mid Z_{i}\right): H^{1}\left(I_{i}, S_{0}\right) \rightarrow$ $H^{1}\left(Z_{i} T\right)$. Let $\alpha$ be the image of the generator of $H^{1}\left(I_{1}, S^{0}\right)$. To prove that $H^{1}(p): H^{1}\left(S^{1}\right) \rightarrow H^{1}(Z)$ is nontrivial, it is enough to prove that $j^{*}(a)$ is nontrivial. Assume that $j^{*}(a)=0$. Then $a=\partial^{*}(d)$ for some $d \in H^{0}(T)$. 
IV.

REMARK. Let $a \in H^{1}(Z, T)$. Assume that $a=\partial^{*}(d)$ for some $d \in H^{0}(T)$, where $\partial^{*}: H^{0}(T) \rightarrow H^{1}(Z, T)$ is the coboundary operator. Then there exists a covering $\mathcal{V}$ of $Z$ such that

(i) $d=j_{\mathcal{V}}^{*}\left(d_{\nu}\right)$ for some $d \nu \in H^{0}(N(\mathcal{V} \mid T))$,

(ii) $a=j_{\mathcal{V}}^{*}(a \mathcal{V})$ for some $a_{\mathcal{V}} \in H^{1}(N(\mathcal{V}), N(\mathcal{V} \mid T))$,

(iii) $\partial^{*}(d \nu)=a v$, where

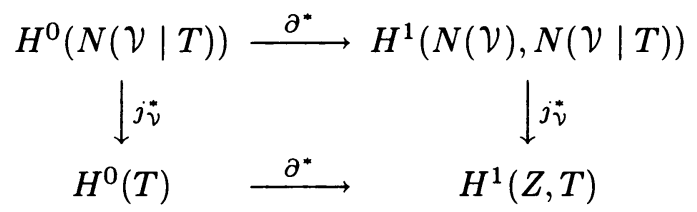

and the $\partial^{*}$ are coboundary operators and the $j_{\mathcal{V}}^{*}$ are natural projections.

V. System of neighborhoods. By $\left\{U_{\alpha}\right\}_{\alpha \in \Lambda}$ we denote the system of all open symmetric (i.e., $s(U) \in \mathcal{U}_{\alpha}$ if $U \in \mathcal{U}_{\alpha}$ ) coverings of $Y$ of order 1. For any covering $\mathcal{U}_{\alpha}$ of $Y$ we define a system $\left\{\mathcal{V}_{\varphi}\right\}_{\varphi \in \Phi_{\alpha}}$ by

$$
\mathcal{V}_{\varphi}=\left\{C^{\prime} \times U \mid U \in \mathcal{U}_{\alpha} \text { and } C^{\prime} \in \mathcal{C}_{\varphi(U)}\right\},
$$

where $\Phi_{\alpha}$ is the set of all symmetric functions $\varphi$ (i.e., $\varphi(U)=\varphi(s(U))$ ) from $U_{\alpha}$ into the set of all nonnegative integers. Let $\Phi=\bigcup\left\{\Phi_{\alpha} \mid \alpha \in \Lambda\right\}$. The system $\left\{U_{\alpha}\right\}_{\alpha \in \Lambda}$ is cofinal in the system of all open coverings of $Y$ and the system $\left\{\mathcal{V}_{\varphi}\right\}_{\varphi \in \Phi}$ is cofinal in the system of all open coverings of $C \times Y$.

Now, let $A$ be any closed subset of $Y$. Suppose that the nerve $N(\mathcal{V})$ is a component of the nerve $N\left(\mathcal{V}_{\varphi} \mid C \times A\right), \varphi \in \Phi_{\alpha}$. Then there is a $k$ an $C^{0} \in C_{k}$ and a subfamily $\mathcal{U}^{0} \subset \mathcal{U}_{\alpha}$ such that

$$
\mathcal{V}=\left\{C^{\prime} \times U \mid U \in \mathcal{U}^{0}, C^{\prime} \in \mathcal{C}_{\varphi(U)}, \text { and } C^{\prime} \subset C^{0}\right\},
$$

so $\bigcup \mathcal{V}=\bigcup \mathcal{U}^{0} \times C^{0}$. Let us observe that

$$
h(\mathcal{V})=\left\{C^{\prime} \times U \mid U \in \mathcal{U}^{0}, C^{\prime} \in C_{\varphi(U)} \text {, and } C^{\prime} \subset h\left(C^{0}\right)\right\}
$$

and $N(h(\mathcal{V}))$ is a component of $N\left(\mathcal{V}_{\varphi} \mid C \times A\right)$ which we denote by $h N(\mathcal{V})$.

VI. Lemma. Let $\mathcal{V}_{\varphi}$ be a covering of $C \times Y, \varphi \in \Phi_{\alpha}$, induced by $\mathcal{U}_{\alpha}$ (see $\S \mathrm{V}$ ). Symmetric components (with respect to $s$ ) of the simplicial complex

$$
L=N\left(\mathcal{V}_{\varphi}\right)-N\left(\mathcal{V}_{\varphi} \mid C \times B\right)
$$

"join" $C \times B_{0}$ and $C \times B_{1}$. By $\Sigma_{i}, i=0,1$, we denote the set of all 1-simplexes that have a vertex in $N\left(\mathcal{V}_{\varphi} \mid C \times B_{i}\right)$ and that are in symmetric components of $L$. We can consider the group $G_{i}$ of all functions from $\Sigma_{i}$ into the integer group as a subgroup (by natural embedding) of $H^{1}\left(N\left(\mathcal{V}_{\varphi}\right), N\left(\mathcal{V}_{\varphi} \mid C \times B\right)\right.$ ).

Let us denote by $\Delta_{i}, i=0,1$, the set of all components of $N\left(\mathcal{V}_{\varphi} \mid C \times B_{i}\right)$. We can consider the group $H^{0}\left(N\left(\mathcal{V}_{\varphi} \mid C \times B_{i}\right)\right)$ as the group of all functions from $\Delta_{i}$ into the integer group. By $\Delta_{i}^{\prime}$ we denote the set of components of $N\left(\mathcal{V}_{\varphi} \mid C \times B_{i}\right)$ that contains a vertex of some simplex of $\Sigma_{i}$. Let $\Delta_{i}^{\prime \prime}=\Delta_{i}-\Delta_{i}^{\prime}$. The group of all functions from $\Delta_{i}^{\prime}$ (resp. $\Delta_{i}^{\prime \prime}$ ) into the integer group we denote by $H_{i}^{\prime}$ (resp. $H_{i}^{\prime \prime}$ ). Let us observe that $\partial^{*}\left(H^{0}\left(N\left(\mathcal{V}_{\alpha} \mid C \times B_{i}\right)\right)\right)=\partial^{*}\left(H_{i}^{\prime}\right) \subset G_{i}$ and $\partial \omega^{*}\left(H_{i}^{\prime \prime}\right)=0$, where

$$
\partial^{*}: H^{0}\left(N\left(\mathcal{V}_{\varphi} \mid C \times B\right)\right) \rightarrow H^{1}\left(N\left(\mathcal{V}_{\varphi}\right), N\left(\mathcal{V}_{\varphi} \mid C \times B\right)\right)
$$


is the coboundary operator. The group $H^{0}\left(N\left(\mathcal{V}_{\varphi} \mid C \times B\right)\right.$ is the direct sum

$$
H^{0}\left(N\left(\mathcal{V}_{\varphi} \mid C \times B_{0}\right)\right) \oplus H^{0}\left(N\left(\mathcal{V}_{\varphi} \mid C \times B_{1}\right)\right) .
$$

The map $s: C \times Y \rightarrow C \times Y$, given by $s(c, y)=(c, s(y))$, induces a symmetric isomorphism

$$
H^{0}(s): H^{0}\left(N\left(\mathcal{V}_{\varphi} \mid C \times B\right)\right) \rightarrow H^{0}\left(N\left(\mathcal{V}_{\varphi} \mid C \times B\right)\right)
$$

in the following way. Any element $d \in H^{0}\left(N\left(\mathcal{V}_{\alpha} \mid C \times B\right)\right)$ is represented by a function $d$ from $\Delta=\Delta_{0} \cup \Delta_{1}$ into the integer group. The element $H^{0}(s)(d)$ can be represented as a map from $\Delta$ into the integer group such that $H^{0}(s)(d)(K)=$ $d(s(K))$ for any component $K$ of $N\left(\mathcal{V}_{\varphi} \mid C \times B\right)$. We have

$$
H^{0}(s)\left(H^{0}\left(N\left(\mathcal{V}_{\varphi} \mid C \times B_{0}\right)\right)\right)=H^{0}\left(N\left(\mathcal{V}_{\varphi} \mid C \times B_{1}\right)\right) .
$$

Now, let $d=d_{0}+d_{1}$ be an element of $H^{0}\left(N\left(\mathcal{V}_{\varphi} \mid C \times B\right)\right.$, where

$$
d_{i} \in H^{0}\left(N\left(\mathcal{V}_{\varphi} \mid C \times B_{i}\right)\right) .
$$

Each $d_{i}$ is represented by a map $d_{i}$ from $\Delta_{i}$ into the integer group. Suppose that $\delta^{*}(d)=0$. Let $\partial_{0} \in \Delta_{0}^{\prime}$ and $\partial_{1}=s\left(\partial_{0}\right) \in \Delta_{1}^{\prime}$. Then there is a (symmetric) simplicial path in $N\left(\mathcal{V}_{\varphi}\right)$ with end points $V_{0} \in \partial_{0}$ and $V_{1}=s\left(V_{0}\right) \in \partial_{1}$ such that all other simplexes of this path are in $L$. Since $\partial^{*}(d)=0$ it follows that $\partial^{*}\left(d_{0}\right)$ and $\partial^{*}\left(d_{1}\right)$ take opposite values on the first and the last simplex of this path. So $d_{0}$ and $d_{1}$ take the same value on $V_{0}$ and $V_{1}$, respectively, and so on $\partial_{0}$ and $\partial_{1}$, respectively. So we obtain the following

LEMMA. Let $d=d_{0}+d_{1}$, where $d_{i} \in H^{0}\left(N\left(\mathcal{V}_{\varphi} \mid C \times B_{i}\right), i=0,1\right.$. If $\partial^{*}(d)=0$, then $d_{0}(K)=d_{1}(s(K))$ for every $K \in \Delta_{0}^{\prime}$ (and so for every $\left.K \in \Delta_{1}^{\prime}\right)$.

VII. Notation. We consider $(Z, T)$ as the sum $\left(Z_{1}, T\right) \cup\left(Z_{2}, T\right)$ of two copies of $(C \times Y, C \times B)$, where $Z_{1} \cap Z_{2}=T$. Let $j_{1}:(C \times Y, C \times B) \rightarrow(Z, T)$ and $j_{2}:(C \times Y, C \times B) \rightarrow(Z, T)$ be corresponding embeddings, so

$$
j_{i}(C \times Y, C \times B)=\left(Z_{i}, T\right) \text { for } i=1,2 .
$$

We may assume that

$$
j_{1}\left|C \times B_{1}=j_{2}\right| C \times B_{1} \text { and } j_{1}\left|C \times B_{0}=j_{2} h\right| C \times B_{0},
$$

where $h: C \times Y \rightarrow C \times Y$ is the homeomorphism given by $h(c, y)=(h(c), y)$ for any $c \in C$ and $y \in Y$. Let $\mathcal{V}_{\varphi}$ be the covering of $C \times Y$ described in $V$. By $\widetilde{\mathcal{V}}_{\varphi}$ we denote the open covering of $Z$ that satisfies two conditions:

$$
j_{1}\left(\mathcal{V}_{\varphi}\right)=\tilde{\mathcal{V}}_{\varphi} \cap Z_{1} \text { and } j_{2}\left(\mathcal{V}_{\varphi}\right)=\tilde{\mathcal{V}}_{\varphi} \cap Z_{2}
$$

Let us observe that the embeddings $j_{1}$ and $j_{2}$ induce isomorphisms of complexes

$$
j_{i}:\left(N\left(\mathcal{V}_{\varphi}\right), N\left(\mathcal{V}_{\varphi} \mid C \times B\right) \rightarrow\left(N\left(\tilde{\mathcal{V}}_{\varphi} \mid Z_{i}\right), N\left(\tilde{\mathcal{V}}_{\varphi} \mid T\right)\right) \quad \text { for } i=1,2\right.
$$

The system of all such coverings $\left\{\tilde{\mathcal{V}}_{\varphi}\right\}_{\alpha \in \Phi}$ of $Z$ is cofinal in the system of all open coverings of $Z$. 
VIII. We have assumed (§III) that $a \in \partial^{*}\left(H^{0}(T)\right)$ i.e. $a=\partial^{*}(d)$ for some $d \in H^{0}(T)$. By the Remark (§IV) there exists a covering $\tilde{\mathcal{V}}_{\varphi}$ of $Z$ that satisfies the conditions (i), (ii), (iii). We have

$$
\begin{aligned}
H^{0}\left(N\left(\widetilde{\mathcal{V}}_{\varphi} \mid T\right)\right) & \stackrel{\partial^{*}}{\rightarrow} H^{1}\left(N\left(\tilde{\mathcal{V}}_{\varphi}\right), N\left(\tilde{\mathcal{V}}_{\varphi} \mid T\right)\right) \\
& =H^{1}\left(N\left(\tilde{\mathcal{V}}_{\varphi} \mid Z_{1}\right), N\left(\widetilde{\mathcal{V}}_{\varphi} \mid T\right)\right) \oplus H^{1}\left(N\left(\tilde{\mathcal{V}}_{\varphi} \mid Z_{2}\right), N\left(\tilde{\mathcal{V}}_{\varphi} \mid T\right)\right)
\end{aligned}
$$

So there exists $a_{\nu} \in H^{1}\left(N\left(\tilde{\mathcal{V}}_{\varphi}\right), N\left(\tilde{\mathcal{V}}_{\varphi} \mid T\right)\right.$ and $d_{\nu} \in H^{0}\left(N\left(\tilde{\mathcal{V}}_{\varphi} \mid T\right)\right)$ such that $a$ is the image of $a_{\nu}$ under $j_{\nu}^{*}$ and $a_{\nu}=\partial^{*}\left(d_{\nu}\right)$. We may assume (see §III) that

$$
a_{\nu} \in H^{1}\left(N\left(\tilde{\mathcal{V}}_{\varphi} \mid Z_{1}\right), N\left(\tilde{\mathcal{V}}_{\varphi} \mid T\right)\right) \subset H^{1}\left(N\left(\tilde{\mathcal{V}}_{\varphi}\right), N\left(\tilde{\mathcal{V}}_{\varphi} \mid T\right)\right)
$$

The embeddings $j_{1}$ and $j_{2}$ induce isomorphisms

$$
H^{1}\left(j_{i}\right): H^{1}\left(N\left(\tilde{\mathcal{V}}_{\varphi} \mid Z_{i}\right), N\left(\tilde{\mathcal{V}}_{\varphi} \mid T\right)\right) \rightarrow H^{1}\left(N\left(\mathcal{V}_{\varphi}\right), N\left(\mathcal{V}_{\varphi} \mid C \times B\right)\right) \quad \text { for } i=1,2
$$

and isomorphisms

$$
H^{0}\left(j_{i}\right): H^{0}\left(N\left(\tilde{\mathcal{V}}_{\varphi} \mid T\right)\right) \rightarrow H^{0}\left(N\left(\mathcal{V}_{\varphi} \mid C \times B\right)\right) \quad \text { for } i=1,2 .
$$

We know that

$$
H^{0}\left(N\left(\tilde{\mathcal{V}}_{\varphi} \mid T\right)\right)=H^{0}\left(N\left(\tilde{\mathcal{V}}_{\varphi} \mid T_{0}\right)\right) \oplus H^{0}\left(N\left(\tilde{\mathcal{V}}_{\varphi} \mid T_{1}\right)\right)
$$

and

$$
H^{0}\left(N\left(\mathcal{V}_{\varphi} \mid C \times B\right)\right)=H^{0}\left(N\left(\mathcal{V}_{\varphi} \mid C \times B_{0}\right)\right) \oplus H^{0}\left(N\left(\mathcal{V}_{\varphi} \mid C \times B_{1}\right)\right)
$$

and that $H^{0}\left(j_{i}\right)$ maps $H^{0}\left(N\left(\widetilde{\mathcal{V}}_{\varphi} \mid T_{0}\right)\right)$ onto $H^{0}\left(N\left(\mathcal{V}_{\varphi} \mid C \times B_{0}\right)\right)$ and $H^{0}\left(N\left(\widetilde{\mathcal{V}}_{\varphi} \mid T_{1}\right)\right)$ onto $H^{0}\left(N\left(\mathcal{V}_{\varphi} \mid C \times B_{1}\right)\right)$.

Let us observe that

$$
H^{0}\left(j_{1}\right)\left|H^{0}\left(N\left(\tilde{\mathcal{V}}_{\varphi} \mid T_{1}\right)\right)=H^{0}\left(j_{2}\right)\right| H^{0}\left(N\left(\tilde{\mathcal{V}}_{\varphi} \mid T_{1}\right)\right)
$$

and

$$
H^{0}\left(j_{1}\right)\left|H^{0}\left(N\left(\tilde{\mathcal{V}}_{\varphi} \mid T_{0}\right)\right)=H^{0}(h) H^{0}\left(j_{2}\right)\right| H^{0}\left(N\left(\tilde{\mathcal{V}}_{\varphi} \mid T_{0}\right)\right)
$$

where $H^{0}(h)$ is an automorphism on $H^{0}\left(N\left(\mathcal{V}_{\varphi} \mid C \times B_{0}\right)\right)$ induced by $h: C \times Y \rightarrow$ $C \times Y$.

Let $d_{\nu}=d_{\nu, 0}+d_{\nu, 1}$ where $d_{\nu, i} \in H^{0}\left(N\left(\tilde{\mathcal{V}}_{\varphi} \mid T_{i}\right)\right)$, for $i=0,1$. Then

$$
H^{0}\left(j_{1}\right)\left(d_{\nu, 0}\right)=H^{0}(h) H^{0}\left(j_{2}\right)\left(d_{\nu, 0}\right)=H^{0}(h)\left(d_{0}\right),
$$

where $d_{0}=H^{0}\left(j_{2}\right)\left(d_{\nu, 0}\right)$ and

$$
H^{0}\left(j_{1}\right)\left(d_{\nu, 1}\right)=H^{0}\left(j_{2}\right)\left(d_{\nu, 1}\right)=d_{1}
$$

We can assume (see $§ I I I)$ that

$$
H^{1}\left(j_{1}\right)\left(a_{\nu}\right) \in G_{0} \subset H^{1}\left(N\left(\mathcal{V}_{\varphi}\right), N\left(\mathcal{V}_{\varphi} \mid C \times B\right)\right)
$$

may be represented as a function that takes the value 1 on each 1-simplex of $\Sigma_{0}$ and that

$$
H^{1}\left(j_{2}\right)\left(a_{\nu}\right) \in H^{1}\left(N\left(\mathcal{V}_{\varphi}\right), N\left(\mathcal{V}_{\varphi} \mid C \times B\right)\right)
$$

is trivial. Let us denote by $d^{1}$ an element of $H^{0}\left(N\left(\mathcal{V}_{\varphi} \mid C \times B_{0}\right)\right)$ that takes the value 1 on each component $K$ of $N\left(\mathcal{V}_{\varphi} \mid C \times B_{0}\right)$.

We have

$$
\partial^{*}\left(H^{0}\left(j_{1}\right)\left(d_{\nu}\right)\right)=H^{1}\left(j_{1}\right)\left(a_{\nu}\right)=\partial^{*}\left(d^{1}\right)
$$


and

$$
\partial^{*}\left(H^{0}\left(j_{2}\right)\left(d_{\nu}\right)\right)=H^{1}\left(j_{2}\right)\left(a_{\nu}\right)=0
$$

It follows that

$$
\partial^{*}\left(H^{0}(h)\left(d_{0}\right)+d_{1}\right)=\partial^{*}\left(d^{1}\right) \text { and } \partial^{*}\left(d_{0}+d_{1}\right)=0 .
$$

by the Lemma in $\S \mathrm{VI}$,

$$
\left(H^{0}(h)\left(d_{0}\right)-d^{1}\right)(K)=d_{1}(s K) \text { and } \quad d_{0}(K)=d_{1}(s K)
$$

for every $K \in \Delta_{0}^{\prime}$. So

$$
d_{0}(K)=\left(H^{0}(h)\left(d_{0}\right)-d^{1}\right)(K)=d_{0}(K)-1 \text { for every } K \in \Delta_{0}^{\prime} .
$$

By $\S \mathrm{V}$ there is a sequence in $\Delta_{0}^{\prime}$ :

$$
K_{1}, K_{2}, \ldots, K_{2^{k}}
$$

such that $K_{n}=h\left(K_{n-1}\right)$ for each $n=2, \ldots, 2^{2 k}$ and $K_{1}=h\left(K_{2^{k}}\right)$. The equality (*) considered on the components $(* *)$ gives a contradiction. Thus $a_{\nu}$ is not in $\partial^{*}\left(H^{0}\left(\widetilde{\mathcal{V}}_{\varphi} \mid T\right)\right)$ and so $a$ is not in $\partial^{*}\left(H^{0}(T)\right)$. It follows that $H^{1}(p): H^{1}\left(S^{1}\right) \rightarrow$ $H^{1}(Z)$ is not trivial.

IX. Let $D$ be the dyadic solenoid, i.e., $D=\operatorname{inv} \lim \left(S^{1} \stackrel{2}{\leftarrow} S^{1} \stackrel{2}{\leftarrow} \cdots\right)$ and let $\pi: D \rightarrow S^{1}$ be the projection onto the first circle.

LEMMA. Let $g$ be a map from a topological space $Z$ into $D$. If $f=\pi \cdot g: Z \rightarrow S^{1}$ is essential then, $Z$ is not movable.

ProOF. Since $\pi: D \rightarrow S^{1}$ is divisible by $2^{n}$ for all $n$ in the group $\left[D, S^{1}\right], f$ is divisible by $2^{n}$ for all $n$ in the group $\left[Z, S^{1}\right]$.

Now let $\left\{Z_{\lambda}, r_{\lambda, \mu}\right\}$ be the Čech system of $Z$ and let $f_{\lambda}: Z_{\lambda} \rightarrow S^{1}$ be a map such that $f \approx f_{\lambda} \pi_{\lambda}, \pi_{\lambda}: Z \rightarrow Z_{\lambda}$ being the projection. Suppose $Z$ is movable, so there is $\mu>\lambda$ such that for arbitrary $\gamma>\mu$ there is a map $h: Z_{\mu} \rightarrow Z_{\gamma}$ with $r_{\lambda \gamma} \cdot h \approx r_{\lambda \mu}$.

Observe that $\left[Z_{\mu}, S^{1}\right]$ does not contain nontrivial elements divisible by all the powers of 2. This is so because such a map induces the trivial homomorphism of fundamental groups. Let $m$ be an integer such that $\left[f_{\lambda} \cdot r_{\lambda \mu}\right]$ is not divisible by $2^{m}$. Since $\left[Z, S^{1}\right]=\operatorname{dir} \lim \left[Z_{\gamma}, S^{1}\right]$, there is $\gamma>\mu$ with $\left[f_{\lambda} \cdot r_{\lambda \gamma}\right]$ divisible by $2^{m}$. Then $\left[f_{\lambda} \cdot r_{\lambda \gamma} \cdot h\right]=\left[f_{\lambda} \cdot r_{\lambda \mu}\right]$ is divisible by $2^{m}$, a contradiction.

REMARK. In the previous Lemma, $D$ can be replaced by any nontrivial solenoid.

X. Solenoidal Roy's example. In [5], P. Roy has given an example of a metric space $X$ with the covering dimension $\operatorname{dim} X=1$ and with the small inductive dimension ind $X=0$. Our solenoidal space $Z$ obtained from the Roy's examples is not movable and it is easy to see that ind $Z=0$. Thus we have the following

THEOREM. There exists a nonmovable 1-dimensional complete metric space $Z$, with ind $Z=0$.

COROLLARY. There exists a nonmovable space $Z$ with movable components (each components is a point).

REMARK. If the covering dimension of a space is 0 , then it is movable. (See [2].) 
XI. Second example. Let $D$ be the dyadic solenoid, i.e.

$$
D=\operatorname{inv} \lim \left(S^{1} \stackrel{2}{\leftarrow} S^{1} \stackrel{2}{\leftarrow} \cdots\right) \text {. }
$$

Let $\pi: D \rightarrow S^{1}$ be the projection onto the first circle. The crucial property of $\pi$ we are going to use is that $\pi$ is essential (not homotopic to a constant map).

Consider the family $\mathbf{A}$ of all open subsets $U$ of $D \times C, C$ being the Cantor set, such that the composition $U \rightarrow D \times C \rightarrow D \rightarrow S^{1}$ is inessential, where $U \rightarrow D \times C$ is the inclusion map, $D \times C \rightarrow D$ is the projection and $D \rightarrow S^{1}$ is $\pi$. Choose a bijection $\alpha: C \rightarrow \mathbf{A}$ between $\mathbf{A}$ and the Cantor set $C$. Such a bijection exists because given a point $(d, c)$ in $D \times C$ there is $\varepsilon>0$ such that the ball $B((d, c), \varepsilon)$ centered at $(d, c)$ and of radius $\varepsilon$ belongs to $\mathbf{A}$. Hence $B((d, c), \delta) \in \mathbf{A}$ for all $\delta<\varepsilon$, and since $D$ is connected $B((d, c), \delta) \neq B((d, c), \mu)$ for $\delta \neq \mu$ sufficiently small. Given $c \in C$, the composition $D \times\{c\} \rightarrow D \times C \rightarrow D \rightarrow S^{1}$ is essential. Therefore, $D \times\{c\}-\alpha(c) \neq \varnothing$ and we can choose $\beta(c) \in D \times\{c\}-\alpha(c)$. Let $Y=\{\beta(c): c \in C\} \subset D \times C$. Observe that $Y \rightarrow D \times C \rightarrow C$ is a bijection $(D \times C \rightarrow C$ being the projection), so $Y$ is totally disconnected. Suppose $\pi \cdot p \mid Y: Y \rightarrow S^{1}$ is inessential, where $p: D \times C \rightarrow D$ is the projection. Then there is a map $H: D \times C \times\{0,1\} \cup Y \times[0,1] \rightarrow S^{1}$ such that $H \mid D \times C \times\{0\}$ is $\pi \cdot p$ and $H \mid D \times C \times\{1\}$ is a constant map. By [7, p. 107], $H$ is homotopic to a map $G$ extendable over a neighborhood $V$ of $D \times C \times\{0,1\} \cup Y \times[0,1]$ in $D \times C \times[0,1]$. Choose a neighborhood $U$ of $Y$ in $D \times C$ such that $U \times[0,1] \subset V$. Then $\pi \cdot p \mid U$ is inessential, so $U=\alpha(c)$ for some $c \in C$. This leads to a contradiction because $Y \cap(D \times\{c\}-\alpha(c)) \neq \varnothing$, so it is impossible for $Y$ to be contained in $U$. Thus $f=\pi \cdot p \mid Y: Y \rightarrow S^{1}$ is essential. By $\S \mathrm{IX}, Y$ is nonmovable.

\section{BIBLIOGRAPHY}

1. K. Borsuk, Theory of shape, Monografie Mat., vol. 50, PWN, Warszawa, 1975.

2. G. Kozlowski and J. Segal, On the shape of 0-dimensional paracompacta, Fund. Math. 83 (1974), 151-154.

3. S. Mardesić and J. Segal, Shape theory, North-Holland, Amsterdam, 1982.

4. M. A. Moron, Prabir Roy's space $\Delta$ as a counterexample in shape theory, Proc. Amer. Math. Soc. (to appear).

5. P. Roy, Failure of equivalence of dimension concepts for metric spaces, Bull. Amer. Math. Soc. 68 (1962), 609-613.

6. E. Spanier, Algebraic topology, McGraw-Hill, New York, 1966.

7. J. J. Walsh, Dimension, cohomological dimension, and cell-like mappings, Shape Theory and Geometric Topology, (Proc., Dubrovnik, 1981), Lecture Notes in Math., vol. 870, SpringerVerlag, Berlin and New York, 1981, pp. 105-118.

Department of Mathematics, University of Tennessee, Knoxville, TenNESSEE 37996

Department of Mathematics, University of Washington, Seattle, WashingTON 98195

Institute of Mathematics PAN, Sniadeckich 8, WaRsaw, Poland 